\title{
SPECTRAL STABILITY OF DEEP TWO-DIMENSIONAL GRAVITY WATER WAVES: REPEATED EIGENVALUES*
}

\author{
BENJAMIN AKERS ${ }^{\dagger}$ AND DAVID P. NICHOLLS ${ }^{\ddagger}$
}

\begin{abstract}
The spectral stability problem for periodic traveling waves on a two-dimensional fluid of infinite depth is investigated via a perturbative approach, computing the spectrum as a function of the wave amplitude beginning with a flat surface. We generalize our previous results by considering the crucially important situation of eigenvalues with multiplicity greater than one (focusing on the generic case of multiplicity two) in the flat water configuration. We use this extended method of transformed field expansions (which now accounts for the resonant spectrum) to numerically simulate the evolution of the eigenvalues as the wave amplitude is increased. We observe that there are no instabilities that are analytically connected to the flat state: The spectrum loses its analyticity at the Benjamin-Feir threshold. We complement the numerical results with an explicit calculation of the first nonzero correction to the linear spectrum of resonant deep water waves. Two countably infinite families of collisions of eigenvalues with opposite Krein signature which do not lead to instability are presented.
\end{abstract}

Key words. spectrum, waves, resonance

AMS subject classifications. 76E17, 76B15, 65N25

DOI. $10.1137 / 110832446$

1. Introduction. From tsunami propagation and the motion of sandbars, to the design of open-ocean oil rigs and pollutant transport, the water wave equations are a central model in fluid mechanics. Among the many motions permitted by these equations, the traveling wave solutions are of great interest due to their ability to transport energy and momentum over great distances in the ocean. These have been studied for over a century, most famously by Stokes, for whom weakly nonlinear periodic waves are now named [1]. In his 1847 paper, Stokes expanded the wave profile as a power series in a small wave slope parameter, a technique which has since become commonplace. This classic Stokes expansion has been applied to the water wave problem numerous times (see, e.g., $[2,3,4,5,6,7]$ ). In this work, we construct a new numerical method, as well as explicit asymptotics, using a similar expansion in the water wave stability problem.

*Received by the editors April 29, 2011; accepted for publication (in revised form) February 24, 2012; published electronically April 24, 2012. This work was performed by an employee of the U.S. Government or under U.S. Government contract. The U.S. Government retains a nonexclusive, royalty-free license to publish or reproduce the published form of this contribution, or allow others to do so, for U.S. Government purposes. Copyright is owned by SIAM to the extent not limited by these rights. Neither the U.S. Government nor any agency thereof, nor any of their employees, make any warranty, express or implied, or assumes any legal liability or responsibility for the accuracy, completeness, or usefulness of any information, apparatus, product, or process disclosed, or represents that its use would not infringe privately owned rights. Reference herein to any specific commercial product, process, or service by trade name, trademark, manufacturer, or otherwise does not necessarily constitute or imply its endorsement, recommendation, or favoring by the U.S. Government or any agency thereof. The views and opinions of authors expressed herein do not necessarily state or reflect those of the U.S. Government or any agency thereof.

http://www.siam.org/journals/siap/72-2/83244.html

$\dagger$ Department of Mathematics and Statistics, Air Force Institute of Technology, 2950 Hobson Way, Wright-Patterson Air Force Base, OH 45433 (benjamin.akers@afit.edu).

${ }_{\ddagger}^{\ddagger}$ Department of Mathematics, Statistics, and Computer Science, University of Illinois at Chicago, 851 S. Morgan, Chicago, IL 60607 (nicholls@math.uic.edu). This author's work was supported by the National Science Foundation through grant DMS-0810958, and by the Department of Energy under award DE-SC0001549. 
Of course not all of these traveling waveforms are dynamically stable, and it is of crucial importance to identify those that are, as these will be the only ones observed in practice. In a series of recent publications $[8,9,10]$ one of the authors has endeavored to study the spectral stability of periodic traveling water waves on a two-dimensional (one vertical and one horizontal) fluid. Spectral stability refers to the fact that the eigenvalues (spectrum) of the water wave operator linearized about the traveling wave are considered, rather than a full linear or even nonlinear stability analysis. The latter would require bounding generic evolving perturbations by the linearized and full water wave equations, respectively, in appropriate function spaces (see [11] for a full discussion of this point). It is not difficult to show that the trivial waves (those of zero amplitude) are weakly stable - i.e., all eigenvalues are pure imaginary - the interesting question becomes the stability of nontrivial traveling wavetrains.

This author approached the problem from a rather different point of view than the direct method applied by $[12,13]$ and more recently by $[14,15]$ (see also the survey article of [16] for a further description of results along these lines). Rather than simply substituting a computed traveling wave into the linearized water wave problem and appealing to a numerical eigensolver, the author used the fact that traveling waves come in analytic branches [17] to show that, generically, the spectral data can also be parametrized analytically [8]. With this point of view, the author followed the "motion" of the spectrum in the complex plane as a wave height/steepness parameter was increased up until divergence of the method [9].

However, this transformed field expansion (TFE) method was fundamentally limited by the requirement that in the trivial (flat water) configuration the eigenvalues must all be simple. While the case of eigenvalues of higher multiplicity is nongeneric, the work of [18] shows that such a configuration is required for the onset of spectral instability for infinitesimal waves. Their result, which arises from the Hamiltonian structure of the water wave problem [19], states that an eigenvalue of the linearized problem can leave the imaginary axis only after collision with another eigenvalue. Additionally, this second eigenvalue must be of opposite Krein signature, though this is not sufficient, as we demonstrate explicitly later. Our main contribution in this work is to extend the TFE algorithm to this crucially important case of eigenvalues of multiplicity two, and to demonstrate the spectral stability of infinitesimal wavetrains to perturbations of the associated eigenfunctions. For deep water waves on a two-dimensional fluid, the eigenvalues have multiplicity one, two, or four, where the only eigenvalue of multiplicity four is $\lambda_{0}=0$, near which the spectrum is not analytic. Thus, by extending the TFE method to eigenvalues of multiplicity two, this paper completes the treatment of the analytic spectrum.

More specifically, in this paper we construct the spectrum of traveling waves as a function of the wave slope, $\epsilon$. We focus on the resonant case, where the linear spectrum of the flat surface has repeated eigenvalues of multiplicity two. At two critical values of the Bloch parameter $(p=0,1 / 4)$ we not only explicitly compute the first three terms in the perturbation expansion at the resonant eigenvalues, but also numerically simulate the spectrum to very high powers of $\epsilon$. We observe that, for deep water waves on a two-dimensional fluid, no real eigenvalues are connected analytically to the flat free surface; i.e., instabilities either bifurcate at finite wave slope or are not analytic in wave slope.

The perturbative approach of this paper is fundamentally different from the more common direct approach $[14,15,20]$. The classic method is to compute the spectrum with an eigensolver for each wave amplitude. To consider a branch of traveling waves, 
the wave amplitude is then discretized. The idea is to choose a discretization which is fine enough to observe any eigenvalue crossings or bubbles of instability. The approach of this paper avoids this difficulty by computing explicitly the dependence of the spectrum on the wave amplitude. Here the spectrum is a continuous (analytic) function of the wave height/slope, so no crossings or bubbles of instability can be "missed" by too coarse a discretization. Along with the computations of the spectrum itself, this method also reports the disk of analyticity of the spectrum, useful knowledge for those considering how fine an amplitude discretization to employ in the classic approach. In addition to any stability conclusions, the spectrum of a traveling wave is useful information by itself, for example as an initial guess for computing time periodic traveling waves [21].

The paper is organized as follows. In section 2, we present the water wave problem as well as two formulations of the spectral stability problem used for numerical methods. First, the TFE formulation, which forms the basis for the new perturbative numerical method, is presented. A second formulation is also presented, which nonperturbatively calculates the spectrum and serves as a basis for comparison. In section 3, we summarize the perturbation procedure used to compute the spectra with two-dimensional kernels; complete details of the perturbation procedure are given in Appendix A. An exact calculation of the spectrum to $O\left(\epsilon^{2}\right)$ using a Stokes-like expansion about two families of repeated eigenvalues is also presented in this section using the same perturbation procedure. In section 4, we present our numerical results compared to the results of the second-order Stokes expansion of the eigenvalues. Conclusions and future areas of research are in section 5 .

2. Spectral stability of traveling water waves. This paper is concerned with the spectral stability of traveling solutions of the potential flow equations in two dimensions [4]:

$$
\begin{aligned}
\phi_{x x}+\phi_{z z}=0, & z<\epsilon \eta, \\
\phi_{z} \rightarrow 0, & z \rightarrow-\infty, \\
\eta_{t}+\epsilon \eta_{x} \phi_{x}=\phi_{z}, & z=\epsilon \eta, \\
\phi_{t}+\frac{\epsilon}{2}\left(\phi_{x}^{2}+\phi_{z}^{2}\right)+\eta=0, & z=\epsilon \eta .
\end{aligned}
$$

These equations describe the motion of an inviscid incompressible fluid on a flat impermeable bed, undergoing an irrotational motion. System (2.1) has been nondimensionalized as in [22], and we assume that the wave slope, $\epsilon=a / L$, is small $-L$ is a length scale chosen in the nondimensionalization so that the waves have spatial period $2 \pi$. In this initial contribution we focus on the deep water case and neglect surface tension. The potential flow equations support traveling solutions which depend analytically on $\epsilon$ (see [17] and the references therein); moreover, the spectral stability problem has simple eigenvalues and eigenfunctions which also depend analytically on $\epsilon$ [9]. We will use this fact to numerically compute the spectrum of traveling waves as a function of $\epsilon$; more specifically we extend the transformed field expansion (TFE) approach of [7] to the crucial case of eigenvalues with multiplicity greater than one. The numerical method of this paper also provides a natural framework for a proof of analyticity of the spectrum of repeated eigenvalues.

Before describing the TFE approach, we recall an important domain decomposition which can be made for the water wave problem with the introduction of an "artificial boundary." The details of this are provided in [7], but we summarize here for completeness. Our goal is to replace $(2.1 \mathrm{~b})$ with an equivalent condition in the 
near-field; consider (2.1a) and (2.1b),

$$
\begin{aligned}
\phi_{x x}+\phi_{z z} & =0, \quad z<\epsilon \eta, \\
\phi_{z} & \rightarrow 0, \quad z \rightarrow-\infty,
\end{aligned}
$$

and notice that, given a plane $\{z=-a\}\left(-a<-\epsilon|\eta|_{\infty}\right)$, these are equivalent to

$$
\begin{aligned}
& \phi_{x x}+\phi_{z z}=0, \quad-a<z<\epsilon \eta, \\
& \phi_{z}=\mu_{z}, \quad z=-a, \\
& \phi=\mu, \quad z=-a, \\
& \mu_{x x}+\mu_{z z}=0, \quad z<-a, \\
& \mu_{z} \rightarrow 0, \quad z \rightarrow-\infty .
\end{aligned}
$$

If we denote $\phi(x,-a)$ by the variable $\Phi(x)$, then it is clear that $(2.2 \mathrm{c}),(2.2 \mathrm{~d})$, and $(2.2 \mathrm{e})$ have the unique solution

$$
\mu(x, z)=\sum_{k} \hat{\Phi}_{k} e^{|k| z} e^{i k x}
$$

where $\hat{\Phi}_{k}$ is the $k$ th Fourier coefficient of $\Phi(x)$. To close (2.2a) and (2.2b) for $\phi$ alone we simply need to produce $\mu_{z}(x,-a)$, which is delivered by the operator $T$,

$$
T[\Phi(x)]:=\sum_{k}|k| \hat{\Phi}_{k} e^{i k x},
$$

a Fourier multiplier of order one; thus, (2.2b) equivalently reads

$$
\phi_{z}(x,-a)-T \phi(x,-a)=0 .
$$

With this operator, (2.1) can be equivalently restated on the truncated (and finite) domain $\{-a<z<\eta\}$ as

$$
\begin{aligned}
\phi_{x x}+\phi_{z z}=0, & -a<z<\epsilon \eta, \\
\phi_{z}-T \phi=0, & z=-a, \\
\eta_{t}+\epsilon \eta_{x} \phi_{x}=\phi_{z}, & z=\epsilon \eta, \\
\phi_{t}+\frac{\epsilon}{2}\left(\phi_{x}^{2}+\phi_{z}^{2}\right)+\eta=0, & z=\epsilon \eta .
\end{aligned}
$$

To search for traveling waves we move to a reference frame moving uniformly with velocity $c \in \mathbf{R}$ and seek steady solutions. With an abuse of notation, we write $\phi=\phi(x+c t, z)-c x$ (so that $\phi$ is still periodic) and $\eta=\eta(x+c t)$, and notice that waves moving to the right have negative $c$; we thus transform (2.3) into

$$
\begin{aligned}
\phi_{x x}+\phi_{z z}=0, & -a<z<\epsilon \eta, \\
\phi_{z}-T \phi=0, & z=-a, \\
c \eta_{x}+\epsilon \eta_{x} \phi_{x}=\phi_{z}, & z=\epsilon \eta, \\
c \phi_{x}+\frac{\epsilon}{2}\left(\phi_{x}^{2}+\phi_{z}^{2}\right)+\eta=0, & z=\epsilon \eta .
\end{aligned}
$$

Copyright (c) by SIAM. Unauthorized reproduction of this article is prohibited. 
2.1. Transformed field expansions. To summarize the TFE approach to computing traveling water waves, we begin with the domain-flattening change of variables

$$
x^{\prime}=x, \quad z^{\prime}=a\left(\frac{z-\epsilon \eta}{a+\epsilon \eta}\right),
$$

which are known as $\sigma$-coordinates [23] in atmospheric science and as the C-method [24] in the electromagnetic theory of gratings. Defining the transformed potential

$$
u\left(x^{\prime}, z^{\prime}\right):=\phi\left(x^{\prime}, \frac{(a+\epsilon \eta) z^{\prime}}{a}+\epsilon \eta\right),
$$

system (2.4) becomes, upon dropping primes,

$$
\begin{aligned}
u_{x x}+u_{z z}=F(x, z ; u, \epsilon \eta), & & -a<z<0, \\
u_{z}-T u=J(x ; u, \epsilon \eta), & & z=-a, \\
c \eta_{x}-u_{z}=Q(x ; u, \epsilon \eta, c), & & z=0, \\
c u_{x}+\eta=R(x ; u, \epsilon \eta, c), & & z=0,
\end{aligned}
$$

where the precise forms for $F, J, Q$, and $R$ are reported in [7]. For the purposes of our brief explanation, the only salient feature of these inhomogeneities is that if $u=\mathcal{O}(\epsilon)$ (noting that we already have $\epsilon \eta=\mathcal{O}(\epsilon)$ ), then they are $O\left(\epsilon^{2}\right)$. The TFE approach now posits the expansions

$$
\begin{gathered}
c=c(\epsilon)=c_{0}+\sum_{n=1}^{\infty} c_{n} \epsilon^{n}, \quad \epsilon \eta=\epsilon \eta(x ; \epsilon)=\sum_{n=1}^{\infty} \eta_{n}(x) \epsilon^{n}, \\
u=u(x, z ; \epsilon)=\sum_{n=1}^{\infty} u_{n}(x, z) \epsilon^{n},
\end{gathered}
$$

which, a posteriori, can be shown to be strongly convergent [17]. Inserting these forms into (2.5) yields

$$
\begin{aligned}
u_{n, x x}+u_{n, z z}=F_{n}(x, z), & & -a<z<0, \\
u_{n, z}-T u_{n}=J_{n}(x), & & z=-a, \\
c_{0} \eta_{n, x}-u_{n, z}=Q_{n}(x)-c_{n-1} \eta_{1, x}, & & z=0, \\
c_{0} u_{n, x}+\eta_{n}=R_{n}(x)-c_{n-1} u_{1, x}, & & z=0,
\end{aligned}
$$

and the forms for $F_{n}, J_{n}, Q_{n}$, and $R_{n}$ are [7]

$$
\begin{gathered}
F_{n}(x, y)=\operatorname{div}_{x}\left[F_{n}^{(1)}(x, y)\right]+\partial_{y} F_{n}^{(2)}(x, y)+F_{n}^{(3)}(x, y) \\
F_{n}^{(1)}=-\frac{2}{a} \sum_{l=1}^{n-1} \eta_{l} \nabla_{x} u_{n-l}-\frac{1}{a^{2}} \sum_{m=2}^{n-1} \sum_{l=1}^{m-1} \eta_{l} \eta_{m-l} \nabla_{x} u_{n-m} \\
+\frac{a+y}{a} \sum_{l=1}^{n-1} \nabla_{x} \eta_{l} \partial_{y} u_{n-l}+\frac{a+y}{a^{2}} \sum_{m=2}^{n-1} \sum_{l=1}^{m-1} \eta_{l} \nabla_{x} \eta_{m-l} \partial_{y} u_{n-m}
\end{gathered}
$$

Copyright $@$ ( ) by SIAM. Unauthorized reproduction of this article is prohibited. 


$$
\begin{aligned}
F_{n}^{(2)}=\frac{a+y}{a} \sum_{l=1}^{n-1} \nabla_{x} \eta_{l} \cdot \nabla_{x} u_{n-l} & +\frac{a+y}{a^{2}} \sum_{m=2}^{n-1} \sum_{l=1}^{m-1} \eta_{l} \nabla_{x} \eta_{m-l} \cdot \nabla_{x} u_{n-m} \\
& -\frac{(a+y)^{2}}{a^{2}} \sum_{m=2}^{n-1} \sum_{l=1}^{m-1} \nabla_{x} \eta_{l} \cdot \nabla_{x} \eta_{m-l} \partial_{y} u_{n-m}
\end{aligned}
$$

$$
\begin{aligned}
F_{n}^{(3)}=\frac{1}{a} \sum_{l=1}^{n-1} \nabla_{x} \eta_{l} \cdot \nabla_{x} u_{n-l}+\frac{1}{a^{2}} \sum_{m=2}^{n-1} \sum_{l=1}^{m-1} \eta_{l} \nabla_{x} \eta_{m-l} \cdot \nabla_{x} u_{n-m} \\
\quad-\frac{a+y}{a^{2}} \sum_{m=2}^{n-1} \sum_{l=1}^{m-1} \nabla_{x} \eta_{l} \cdot \nabla_{x} \eta_{m-l} \partial_{y} u_{n-m}
\end{aligned}
$$

$$
\begin{aligned}
Q_{n}= & -\sum_{l=1}^{n-2} c_{l} \cdot \nabla_{x} \eta_{n-l}-\frac{1}{a} \sum_{m=1}^{n-1} \sum_{l=0}^{m-1} c_{l} \cdot \eta_{m-l} \nabla_{x} \eta_{n-m}-\sum_{l=1}^{n-1} \nabla_{x} \eta_{l} \cdot \nabla_{x} u_{n-l} \\
& -\frac{1}{a} \sum_{m=2}^{n-1} \sum_{l=1}^{m-1} \eta_{l} \nabla_{x} \eta_{m-l} \cdot \nabla_{x} u_{n-m}+\sum_{m=2}^{n-1} \sum_{l=1}^{m-1} \nabla_{x} \eta_{l} \cdot \nabla_{x} \eta_{m-l} \partial_{y} u_{n-m}
\end{aligned}
$$

$$
\begin{aligned}
& R_{n}=-\sum_{l=1}^{n-2} c_{l} \cdot \nabla_{x} u_{n-l}-\frac{2}{a} \sum_{m=1}^{n-1} \sum_{l=0}^{m-1} c_{l} \cdot \eta_{m-l} \nabla_{x} u_{n-m} \\
& -\frac{1}{a^{2}} \sum_{t=2}^{n-1} \sum_{m=1}^{t-1} \sum_{l=0}^{m-1} c_{l} \cdot \eta_{m-l} \eta_{t-m} \nabla_{x} u_{n-t}+\sum_{m=1}^{n-1} \sum_{l=0}^{m-1} c_{l} \cdot \nabla_{x} \eta_{m-l} \partial_{y} u_{n-m} \\
& +\frac{1}{a} \sum_{t=2}^{n-1} \sum_{m=1}^{t-1} \sum_{l=0}^{m-1} c_{l} \cdot \eta_{m-l} \nabla_{x} \eta_{t-m} \partial_{y} u_{n-t}-\frac{2}{a} g \sum_{l=1}^{n-1} \eta_{l} \eta_{n-l} \\
& \quad+\frac{1}{a} g \sum_{m=2}^{n-1} \sum_{l=1}^{m-1} \eta_{l} \eta_{m-l} \eta_{n-m}+\frac{2}{a} \sigma \sum_{l=1}^{n-1} \eta_{l} \Delta_{x} \eta_{n-l} \\
& -\frac{1}{a} \sum_{m=2}^{n-1} \sum_{m=1}^{n-1} \sum_{l=1}^{m-1} \eta_{l} \eta_{l} \nabla_{m-l} u_{m-l} \cdot \nabla_{x} u_{n-m}-\frac{1}{2 a^{2}} \sum_{t=3}^{n-1} \sum_{m=2}^{t-1} \sum_{l=1}^{m-1} \eta_{l} \eta_{m-l} \nabla_{x} u_{t-m} \cdot \nabla_{x} u_{n-t} \nabla_{x} u_{l} \cdot \nabla_{x} u_{n-l} \\
& +\sum_{m=2}^{n-1} \sum_{l=1}^{m-1} \nabla_{x} \eta_{l} \cdot \nabla_{x} u_{m-l} \partial_{y} u_{n-m}+\frac{1}{a} \sum_{t=3}^{n-1} \sum_{m=2}^{t-1} \sum_{l=1}^{m-1} \eta_{l} \nabla_{x} \eta_{m-l} \cdot \nabla_{x} u_{t-m} \partial_{y} u_{n-t} \\
& -\frac{1}{2} \sum_{t=3}^{n-1} \sum_{m=2}^{t-1} \sum_{l=1}^{m-1} \nabla_{x} \eta_{l} \cdot \nabla_{x} \eta_{m-l} \partial_{y} u_{t-m} \partial_{y} u_{n-t}-\frac{1}{2} \sum_{l=1}^{n-1} \partial_{y} u_{l} \partial_{y} u_{n-l} .
\end{aligned}
$$

Copyright (c) by SIAM. Unauthorized reproduction of this article is prohibited. 
Given a traveling solution of the water wave problem, $(\bar{u}, \bar{\eta}, \bar{c})$, we consider its spectral stability with the ansatz

$$
u(x, z, t)=\bar{u}(x, z)+v(x, z) e^{\lambda t}, \quad \eta(x, t)=\bar{\eta}(x, z)+\zeta(x) e^{\lambda t},
$$

where quadratic products of the perturbations $v$ and $\zeta$ are neglected. Next, the eigenvalues $\lambda$ and the functions $\zeta$ and $v$ are also expanded as a power series in $\epsilon$. Such a procedure in the TFE formulation of the water wave problem yields

$$
\begin{aligned}
v_{n, x x}+v_{n, z z}=\tilde{F}_{n}(x, z), & & -a<z<0, \\
v_{n, z}-T v_{n}=\tilde{J}_{n}(x), & & z=-a, \\
\lambda_{0} \zeta_{n}+c_{0} \zeta_{n, x}-v_{n, z}=\tilde{Q}_{n}(x)-c_{n-1} \eta_{1, x}-\lambda_{n-1} \zeta_{1}, & & z=0, \\
\lambda_{0} \zeta_{n}+c_{0} v_{n, x}+\eta_{n}=\tilde{R}_{n}(x)-c_{n-1} u_{1, x}-\lambda_{n-1} v_{1}, & & z=0 .
\end{aligned}
$$

This procedure is presented in [8], and the functions $\tilde{F}_{n}, \tilde{J}_{n}, \tilde{Q}_{n}$, and $\tilde{R}_{n}$ are known explicitly. The salient feature here is that for $n=0$ these functions vanish. We comment that to consider the most general perturbations possible we enforce "Bloch periodicity" in the $x$ variable for $\zeta$ and $v$. Recall that this requires

$$
\zeta(x+\gamma)=e^{i p \gamma} \zeta(x), \quad v(x+\gamma, z)=e^{i p \gamma} v(x, z) \quad \forall \gamma \in 2 \pi \mathbf{Z},
$$

and allows one to express

$$
\zeta(x)=\sum_{k=-\infty}^{\infty} \hat{\zeta}_{k} e^{i(k+p) x}, \quad v(x, z)=\sum_{k=-\infty}^{\infty} \hat{v}_{k}(z) e^{i(k+p) x}
$$

see $[8,9]$. For each value of $p$, the spectral stability problem is transformed from an eigenvalue problem into a series of linear problems (see (2.8)) for the corrections $v_{n}$ and $\zeta_{n}$. Over the range of the linear operator, the problem is easily solved via Fourier transforms. The key step numerically is solving for the Fourier amplitudes of the frequencies in the null space and the eigenvalue corrections - each from solvability conditions. In the following sections, we present the form of the perturbation procedure and solvability conditions, the resulting numerical method, the exactly calculated first nonzero correction to the spectrum, as well as numerical computations of the spectrum to arbitrary order. To test the numerical computations, we also compute the spectrum nonperturbatively in what we call direct numerical simulation (DNS).

2.2. Direct numerical simulation. In section 4 , we compare numerical results of the TFE method to those of a competing method for calculating the spectrum, which we call DNS. This method is outlined in [10] and uses the Hamiltonian structure of the water wave problem first presented in [19], together with the canonical variables $\eta(x, t)$ and the surface velocity potential

$$
\xi(x, t):=\phi(x, \eta(x, t), t) .
$$

This formulation can be made more explicit [25] with the introduction of the DirichletNeumann operator,

$$
G(\eta) \xi=\left(\partial_{z} \phi-\left(\partial_{x} \eta\right) \partial_{x} \phi\right)_{z=\eta}
$$


which maps Dirichlet data to Neumann data. In terms of this operator the evolution equations (2.1) can be equivalently stated as

$$
\begin{aligned}
& \partial_{t} \eta=G(\eta) \xi \\
& \partial_{t} \xi=-\eta-A(\eta) B(\eta, \xi),
\end{aligned}
$$

where

$$
\begin{aligned}
A(\eta) & =\frac{1}{2\left(1+\left(\partial_{x} \eta\right)^{2}\right)}, \\
B(\eta, \xi) & =\left(\partial_{x} \xi\right)^{2}-(G(\eta) \xi)^{2}-2\left(\partial_{x} \eta\right)\left(\partial_{x} \xi\right) G(\eta) \xi .
\end{aligned}
$$

If $(\bar{\eta}, \bar{\xi}, \bar{c})$ is a steady solution in a frame traveling with velocity $\bar{c}$ (i.e., a traveling wave solution with velocity $\bar{c}$ ), then we can study spectral stability by considering

$$
\eta(x, t)=\bar{\eta}(x)+e^{\lambda t} \zeta(x), \quad \xi(x, t)=\bar{\xi}(x)+e^{\lambda t} \psi(x) .
$$

Upon insertion of these forms into (2.9) (appropriately modified to account for the traveling frame) and ignoring quadratic products, we find the eigenproblem

$$
\begin{aligned}
\left(\lambda+c \partial_{x}\right) \zeta & =G_{\eta}(\bar{\eta})[\bar{\xi}]\{\zeta\}+G(\bar{\eta})[\psi], \\
\left(\lambda+c \partial_{x}\right) \psi & =-\zeta-A_{\eta}(\bar{\eta})\{\zeta\} B(\bar{\eta}, \bar{\xi})-A(\bar{\eta})\left(B_{\eta}(\bar{\eta}, \bar{\xi})\{\zeta\}+B_{\xi}(\bar{\eta}, \bar{\xi})\{\psi\}\right),
\end{aligned}
$$

where $\eta$ and $\xi$ subscripts represent functional variations in $\eta$ and $\xi$, respectively, in the $\zeta$ and $\psi$ directions. For complete details together with a specification of a spectral collocation procedure for estimating the eigenvalues $\lambda$ and eigenfunctions $(\zeta, \psi)$, we refer the interested reader to [10].

3. A perturbation procedure for repeated eigenvalues. The spectral stability problem is a generalized eigenvalue problem of the form

$$
(A+\lambda B) q=0
$$

where the operators $A$ and $B$ depend analytically on $\epsilon$. The approach we advocate is essentially a Rayleigh-Schrödinger expansion (see [26]), which is based upon the assumption that the spectral data $(\lambda, q)$ also vary analytically in $\epsilon$. If this is the case, we can form the Taylor series

$$
A=\sum_{n=0}^{\infty} A_{n} \epsilon^{n}, \quad B=\sum_{n=0}^{\infty} B_{n} \epsilon^{n}, \quad \lambda=\sum_{n=0}^{\infty} \lambda_{n} \epsilon^{n}, \quad q=\sum_{n=0}^{\infty} q_{n} \epsilon^{n},
$$

and upon insertion into (2.5) we find a series of linear problems of the form

$$
\left(A_{0}+\lambda_{0} B_{0}\right) q_{n}=P_{n}
$$

with $P_{n}$ a known function of $\left\{\lambda_{1}, \ldots, \lambda_{n}\right\}$ and $\left\{q_{0}, \ldots, q_{n-1}\right\}$ which vanishes when $n=0$. As we will explain in more detail in this section, although the functional form of $P_{n}$ is known at order $n$, the values of some of its arguments, for example $\lambda_{n}$, are not determined at this order. The eigenvalue problem is solved by inversion of the linear operator $\left(A_{0}+\lambda_{0} B_{0}\right)$ in its range, as well as by solvability conditions which set both the components of $q_{n}$ in the null space of this operator as well as the correction to the eigenvalue $\lambda_{n}$.

Copyright (C) by SIAM. Unauthorized reproduction of this article is prohibited. 
The case where $\lambda_{0}$ is simple is handled in [8] and leads to a strongly convergent Taylor series for $\lambda$ and $q$ which can be used in a stable and high-order numerical simulation of the spectral data $[9,10]$. This algorithm numerically inverts the operator $\left(A_{0}+\lambda_{0} B_{0}\right)$ over its range using a Chebyshev-based elliptic solver in the vertical direction and Fourier collocation in the horizontal dimension. The eigenvalues $\lambda_{n}$ are set by a linear solvability condition at each order, ensuring that the operator is forced orthogonally to its null space.

We now take up the far more challenging question of $\lambda_{0}$ with higher multiplicity, more specifically algebraic and geometric multiplicity two. Suppose that $\left(A_{0}+\lambda_{0} B_{0}\right) q_{0}=0$ has two independent solutions $w_{1}$ and $w_{2}$. At every order, for (3.3) to have a solution one requires that $P_{n}$ be orthogonal to the kernel of the adjoint operator $\left(A_{0}+\lambda_{0} B_{0}\right)^{*}$, which we suppose is spanned by $\psi_{1}$ and $\psi_{2}$. In the simple case $\left.\operatorname{dim}\left(\operatorname{Ker}\left(A_{0}+\lambda_{0} B_{0}\right)^{*}\right)\right)=1$, the solvability condition $\left\langle\psi_{1}, P_{n}\right\rangle=0$ is a single linear equation determining $\lambda_{n}$ [9]. We generalize this procedure to find solutions when the kernel is two-dimensional, using solvability arguments [26, 27].

In more detail, the eigenfunction is most generally represented as an arbitrary linear combination of $w_{1}$ and $w_{2}$,

$$
q_{0}=\alpha w_{1}+\beta w_{2} .
$$

Without loss of generality we choose $\alpha=1$, defining the total projection of $q$ onto $w_{1}$. Although the coefficient $\beta$ is arbitrary at leading order $(n=0)$, a perturbative solution exists only in the neighborhood of particular values of $\beta$. To find these values we expand $\beta$ as a power series in $\epsilon$,

$$
\beta=\sum_{n=0}^{\infty} \beta_{n} \epsilon^{n} .
$$

At each order, two solvability conditions, $\left\langle\psi_{j}, P_{n}\right\rangle=0$, are satisfied by choice of $\lambda_{n}$ and $\beta_{n}$. Thus far the discussion has been quite generic, but, of course, the forms of the equations for $\beta_{n}$ and $\lambda_{n}$ are problem-specific, and for deep water waves, the equations for $\lambda_{n}$ and $\beta_{n}$ are all linear.

We next present the perturbation procedure used to numerically compute $\lambda_{n}$ to all orders. At each order, we wish to solve a linear algebra problem of the form

$$
\left(A_{0}+\lambda_{0} B_{0}\right) q_{n}=-\sum_{j=1}^{n}\left(A_{j}+\sum_{q=0}^{n} \lambda_{q} B_{j-q}\right) q_{n-j} .
$$

The leading-order solution is given by (3.4) and (3.10), with an as yet arbitrary ratio $\beta_{0}$. The ratio $\beta_{0}$ will be determined at a later power of $\epsilon$, with the power depending on the two frequencies in the leading-order solution, $\xi_{1}$ and $\xi_{2}$. At $\mathcal{O}(\epsilon)$, the system is

$$
\left(A_{0}+\lambda_{0} B_{0}\right) q_{1}=-\left(A_{1}+\lambda_{0} B_{1}+\lambda_{1} B_{0}\right) q_{0} .
$$

Taking the inner product of the right-hand side with the $\psi_{j}$ yields two solvability conditions,

$$
\left(\begin{array}{cc}
\left\langle\psi_{1},\left(A_{1}+\lambda_{1} B_{0}+\lambda_{0} B_{1}\right) w_{1}\right\rangle & \left\langle\psi_{1},\left(A_{1}+\lambda_{1} B_{0}+\lambda_{0} B_{1}\right) w_{2}\right\rangle \\
\left\langle\psi_{2},\left(A_{1}+\lambda_{1} B_{0}+\lambda_{0} B_{1}\right) w_{1}\right\rangle & \left\langle\psi_{2},\left(A_{1}+\lambda_{1} B_{0}+\lambda_{0} B_{1}\right) w_{2}\right\rangle
\end{array}\right)\left(\begin{array}{c}
1 \\
\beta_{0}
\end{array}\right)=0 .
$$

Copyright ( $)$ by SIAM. Unauthorized reproduction of this article is prohibited. 
Clearly our procedure depends on whether any of the inner products in (3.6) vanish, which in turn depends on the frequency difference $\xi_{1}-\xi_{2}$. We use the convention that both the $\psi_{j}$ and $w_{j}$ are supported solely at wavenumber $\xi_{j}$. Because the operators $A_{n}$ and $B_{n}$ have support at frequencies $|\xi| \leq n$, the inner products vanish at least when $\left|\xi_{1}-\xi_{2}\right|>n$, that is, if the frequency difference is larger than the order of the perturbation. Inner products may also vanish at order $n$ when $\left|\xi_{1}-\xi_{2}\right| \leq n$ due to cancellation, but for this one must compute the inner products on a case-by-case basis. Next we present the derivation of $\lambda_{1}$ and $\beta_{0}$ for deep water waves, with the complete details of the calculation of all values of $\lambda_{n}$ and $\beta_{n}$ left to Appendix A.

First, the null space is two-dimensional for fixed $p$ if there are two frequencies $\xi_{1}, \xi_{2}$ with $\lambda_{0}\left(\xi_{1}\right)=\lambda_{0}\left(\xi_{2}\right)$. For the water wave problem this is equivalent to

$$
\xi_{1}-\xi_{2}-m=0 \quad \text { and } \quad \omega\left(\xi_{2}\right)+\omega\left(\xi_{1}\right)-m \omega(1)=0,
$$

where $m$ is an integer. To enumerate the possibilities, we note that we can choose $m=1$ only if the linear (two-dimensional) system supports resonant triads, which is not possible for two-dimensional deep gravity water waves [28]. Generally, the system will have a two-dimensional kernel for any wavenumbers $\xi_{1}$ and $\xi_{2}$ which undergo an $(m+2)$ degree resonance with $m$ instances of $\xi=1[18,16]$. The details of the procedure for calculating $\beta_{n}$ and $\lambda_{n}$ depend crucially on the degree of the resonance. Because there are no triads in deep water we know that $\left|\xi_{1}-\xi_{2}\right|>1$, or that

$$
\left\langle\psi_{j},\left(A_{1}+\lambda_{0} B_{1}\right) w_{i}\right\rangle=0
$$

for $i \neq j$. Also, as there is no mean flow,

$$
\left\langle\psi_{i},\left(A_{1}+\lambda_{0} B_{1}\right) w_{i}\right\rangle=0
$$

(here the result would differ for shallow water); thus (3.6) simplifies to

$$
\lambda_{1}\left(\begin{array}{cc}
\left\langle\psi_{1}, B_{0} w_{1}\right\rangle & 0 \\
0 & \left\langle\psi_{2}, B_{0} w_{2}\right\rangle
\end{array}\right)\left(\begin{array}{c}
1 \\
\beta_{0}
\end{array}\right)=0
$$

which implies $\lambda_{1}=0$ with $\beta_{0}$ arbitrary. Continuing this procedure, we find that in deep water $\beta_{0}$ is always zero and is determined at $\mathcal{O}\left(\epsilon^{2}\right)$. Moreover, there are two branches of eigenvalue-eigenfunction pairs which are, at leading order, supported at a single frequency $\xi_{j}$; the coupling of the two frequencies happens only at higher powers in $\epsilon$. Complete details of the perturbation procedure, including which inner products vanish in deep water, are given in Appendix A. Because the procedure is sensitive to which frequencies the operators are supported at, the algorithm will require extension for finite depth, where there is a mean flow. The current algorithm includes support for triads, even though there are none in deep water, so that it may be more easily extended to include surface tension and to shallow water [28].

For deep water gravity waves, the leading-order linear problem is

$$
\begin{aligned}
v_{0, x x}+v_{0, z z} & =0, & & -a<z<0, \\
v_{0, z}-T v_{0} & =0, & & z=-a, \\
\lambda_{0} \zeta_{0}+c_{0} \zeta_{0, x}-v_{0, z} & =0, & & z=0, \\
\lambda_{0} v_{0}+c_{0} v_{0, x}+\zeta_{0} & =0, & & z=0,
\end{aligned}
$$


so that

$$
A_{0}=\left(\begin{array}{cc}
\partial_{x}^{2}+\partial_{z}^{2} & 0 \\
\partial_{z}-T & 0 \\
-\partial_{z} & c_{0} \partial_{x} \\
c_{0} \partial_{x} & 1
\end{array}\right) \quad \text { and } \quad B_{0}=\left(\begin{array}{cc}
0 & 0 \\
0 & 0 \\
0 & 1 \\
1 & 0
\end{array}\right)
$$

The vector $q_{0}$ can be thought of as having two components $q_{0}=\left(v_{0}(x, z), \zeta_{0}(x)\right)$, so long as the operators in the rows of $A_{0}$ are applied at the appropriate places (inside the domain for the first row, at $z=a$ for the second row, etc.). The kernel of $\left(A_{0}+\lambda_{0} B_{0}\right)$ can then be determined by solving the first two equations for the $z$-dependence of $v_{0}$, essentially reducing the problem to surface quantities, or to solving

$$
\left(\begin{array}{cc}
-\left(-\partial_{x}^{2}\right)^{1 / 2} & \lambda_{0}+c_{0} \partial_{x} \\
\lambda_{0}+c_{0} \partial_{x} & 1
\end{array}\right)\left(\begin{array}{c}
v_{0}(x, 0) \\
\zeta_{0}(x)
\end{array}\right)=0 .
$$

It is simple to show that this kernel is supported at frequencies satisfying

$$
\left(\lambda_{0}+i c_{0} \xi\right)^{2}+|\xi|=0
$$

or, using $c_{0}=1$,

$$
\lambda_{0}(k, p)= \pm i \omega(k+p)-i(k+p)
$$

where $\omega(\xi):=\sqrt{|\xi|}, k \in \mathbf{Z}$, and $p \in(-1 / 2,1 / 2]$ is the quasi-period parameter mentioned in section 2 . Because the water wave problem is Hamiltonian with real solutions, it is enough to consider only $p \geq 0$ (the negative $p$ values are determined as the complex conjugate) [11].

At later orders, the differential operator $\left(A_{0}+\lambda_{0} B_{0}\right)$ must be inverted over its range. In the numerical method, given that the frequencies in the null space are known, this is naturally handled in Fourier space, where inverting the operator means solving for all the Fourier modes of the solution except the two in the null space. Since the right-hand side of (2.8) is nonhomogeneous for $n \geq 1$, a Chebyshev-based elliptic solver is used to determine the vertical dependence of the Fourier modes. This inversion of $\left(A_{0}+\lambda_{0} B_{0}\right)$ is identical to the method used for simple eigenvalues, with the exception that there are now two elements which are not in the range of the operator, rather than one. After all the frequencies in the range are determined, the amplitude of the Fourier modes in the null space is determined using the perturbation procedure outlined above. The correction $v_{n}$ has zero projection onto the eigenfunction $w_{1}$ by definition and has a projection onto $w_{2}$ of amplitude $\beta_{n}$ which is determined by a solvability condition. The numerical method requires two loops, one in the perturbation order and another in the wave numbers of the correction $v_{n}$, for each $\lambda_{0}$. The different eigenvalues can then be treated separately, as can the different Bloch parameters - either in other loops or in parallel.

3.1. The $\boldsymbol{O}\left(\epsilon^{2}\right)$ spectrum. An eigenvalue of multiplicity two, where the kernel of $\left(A_{0}-\lambda_{0} B_{0}\right)$ is two-dimensional, requires that for fixed $p$ there be distinct integers $k_{1}$ and $k_{2}$ with $\lambda_{0}\left(k_{1}, p\right)=\lambda_{0}\left(k_{2}, p\right)$. For deep water waves, this happens, for $p \in[0,1 / 2]$, only when $p=0$ and $1 / 4$. Moreover, in the deep water problem, eigenvalues have multiplicity only one, two, or four-as can be seen using the graphic construction for computing wave resonances; see [28]. There is only one eigenvalue of multiplicity four, the degenerate case $\lambda_{0}=0$. Near $\lambda_{0}=0$ the spectrum is not analytic with respect 
to $\epsilon$ due to the Benjamin-Feir instability; thus considering the two-dimensional case completes the treatment of the analytic spectrum. In this section, we compute explicitly the first nonzero corrections for the two countably infinite families of repeated eigenvalues at $p=0$ and $1 / 4$, which we use in section 4 to compare the results of our numerical computations to arbitrary order. To our knowledge, we are the first to calculate the Stokes expansion for these families of eigenvalues.

At $p=0$, there is a countably infinite set of eigenvalues, $\lambda_{0}$, with two distinct frequencies in the null space, $\xi_{1}=k_{1}+p$ and $\xi_{2}=k_{2}+p$, given by

$$
\lambda_{0}=-i n(n+1), \quad \xi_{1}=n^{2}, \quad \text { and } \quad \xi_{2}=(n+1)^{2}
$$

for $n \in \mathbf{N}^{+}$, and, of course, another family given by the complex conjugate. From each of these flat-state eigenvalues, $\lambda_{0}$, we have computed the Stokes expansion, calculating explicitly two branches of eigenvalues, whose eigenfunctions are supported at leading order $\xi_{1}$ and $\xi_{2}$. The eigenvalue corrections are

$$
\lambda_{1}=0, \quad \lambda_{2}^{(1)}=2 i n^{2}, \quad \lambda_{2}^{(2)}=2 i(n+1)^{2},
$$

where the superscript on $\lambda_{2}^{(j)}$ refers to the single frequency $\xi_{j}$ at which the eigenvector is supported at $\mathcal{O}\left(\epsilon^{0}\right)$. For all members of this family of eigenvalues, the frequencies $\xi_{j}$ are at leading order decoupled, $\beta_{0}=0$.

At $p=1 / 4$ there is another countably infinite set of eigenvalues, $\lambda_{0}$, with twodimensional null space,

$$
\lambda_{0}=i\left(\frac{1}{4}-n^{2}\right), \quad \xi_{1}=\frac{(2 n-1)^{2}}{4}, \quad \xi_{2}=\frac{(2 n+1)^{2}}{4}
$$

for all $n \in \mathbf{N}$. A Stokes expansion has also been carried out about this second family of $\lambda_{0}$. The eigenvalue corrections are $\lambda_{1}=0$ and

$$
\lambda_{2}^{(1)}=-i\left(\frac{1}{4}\right), \quad \lambda_{2}^{(2)}=i\left(\frac{9}{2}\right), \quad n=0
$$

and

$$
\lambda_{2}^{(1)}=i\left(\frac{(2 n-1)^{2}}{2}\right), \quad \lambda_{2}^{(2)}=i\left(\frac{(2 n+1)^{2}}{2}\right), \quad n>0 .
$$

As with the previous family of eigenvalues, the set at $p=1 / 4$ also has $\beta_{0}=0$. Notice here that both at $p=0$ and at $p=1 / 4$ the $\lambda_{2}^{(j)}$ are all pure imaginary; thus to $\mathcal{O}\left(\epsilon^{2}\right)$ there are no instabilities from these sets of eigenvalues. In fact, based upon our numerical experiments detailed in section 4, we conjecture that the full Taylor sums are purely imaginary for all real values of $\epsilon$ (within the disk of analyticity).

In both families we have labeled the frequencies using the convention that

$$
\lambda_{0}^{-}\left(\xi_{1}\right)=-\sqrt{\left|p+\xi_{1}\right|}-\left(p+\xi_{1}\right)=\sqrt{\left|p+\xi_{2}\right|}-\left(p+\xi_{2}\right)=\lambda_{0}^{+}\left(\xi_{2}\right) .
$$

The frequency $\xi_{1}$ has $\lambda_{0}$ with negative Krein signature, while $\xi_{2}$ corresponds to $\lambda_{0}$ with positive Krein signature. Both of these families satisfy the necessary, but not sufficient, condition of [18] that only collisions of eigenvalues with opposite Krein signature may lead to instability.

Regarding Krein signature, we recall that the traveling water wave problem may be recast as that of finding critical points of a functional involving the Hamiltonian 
(see [18]), say $H_{c}$. If $\lambda$ is a nonzero, purely imaginary, simple eigenvalue of the problem linearized about the equilibrium, then the second variation of $H_{c}$ at the equilibrium is either positive or negative definite; this sign is the Krein signature of $\lambda$, and it is conserved up to collision with another eigenvalue. The fundamental result of MacKay and Saffman [18] is that if two simple, pure imaginary eigenvalues of the same Krein signature collide at a point other than zero, then they cannot leave the imaginary axis. Thus, for infinitesimal waves, the principal instabilities should arise from eigenvalues of multiplicity larger than one.

The formulae of the previous two paragraphs were computed using a cubic approximation of the water wave equations expanded about the free surface, as in $[29,30]$. Although a nonlocal formulation, this cubic approximation of the potential flow equations should not be confused with that of [31], used for the spectral stability problem by [15]. The cubic truncation is also entirely different from that used in the numerical method, and as such these eigenvalues (3.12), (3.14a), and (3.14b) may be used as a test both of the numerics as well as of the TFE formulation. In particular, the exact values of $\lambda_{2}$ can be used to measure robustness of the TFE formulation for small artificial depth $a$-see [32] for a numerical investigation of this robustness in the traveling wave problem.

4. Numerical results. We have extended the TFE method for perturbatively computing the spectrum of traveling waves to eigenvalues of multiplicity two (the multiplicity one case is handled in [9]). For two-dimensional deep water waves, no eigenvalues both are analytic in $\epsilon$ and have multiplicity higher than two, so the resulting numerical method computes the entire analytic spectrum. The method is implemented in $\mathrm{C}++$, using a Fourier-Chebyshev scheme to discretize (2.8) as well as to evaluate the inner products in section 3 . The resulting eigenvalue problem decouples different values of the Bloch parameter $p$ which can then be treated in parallel. For each value of $p$, the eigenvalues $\lambda(k, p)$ can also be treated in parallel (or sequentially in a loop). For each value of $p$ and $\lambda(k, p)$, the perturbation series is solved for using the procedure of section 3. Because instabilities arise only from repeated eigenvalues, this method allows for significant savings in computing instabilities, as one can restrict the computation to only the repeated eigenvalues.

Here we present the results of the method applied to the deep water case, where we have both explicitly computed the second correction to the eigenvalues $\lambda_{2}$ (cf. (3.12), (3.14a), and (3.14b)) and numerically computed the corrections $\lambda_{n}$ for arbitrary $n$. We compare the performance of a high-order perturbative computation $(n=16)$ to both a low-order perturbative calculation $(n=2)$ and the DNS approach outlined in section 2.2 .

In Figure 4.1 we present three independent computations of the spectrum for traveling waves near the flat surface eigenvalue $\lambda_{0}=6 i$ at $p=0$. The first is an explicit second-order perturbative computation in the potential flow equations, Taylor expanded about $z=0$, as in [30, 29], whose formula may be recovered by taking the complex conjugate of (3.13). The second computation is our new numerical procedure outlined in section 3 , taken to perturbation order $n=16$. The third set of data is generated by a spectral collocation implementation of the DNS method outlined in section 2.2. In Figure 4.1(left) we observe that the $\mathcal{O}\left(\epsilon^{2}\right)$ computation well approximates the entire series for small $\epsilon$. From the right panel, we observe that only a few more terms $n \approx 16$ are necessary to resolve larger amplitudes to the precision of our DNS.

In Figure 4.2, the convergence rate of the TFE method is observed. The eigen- 

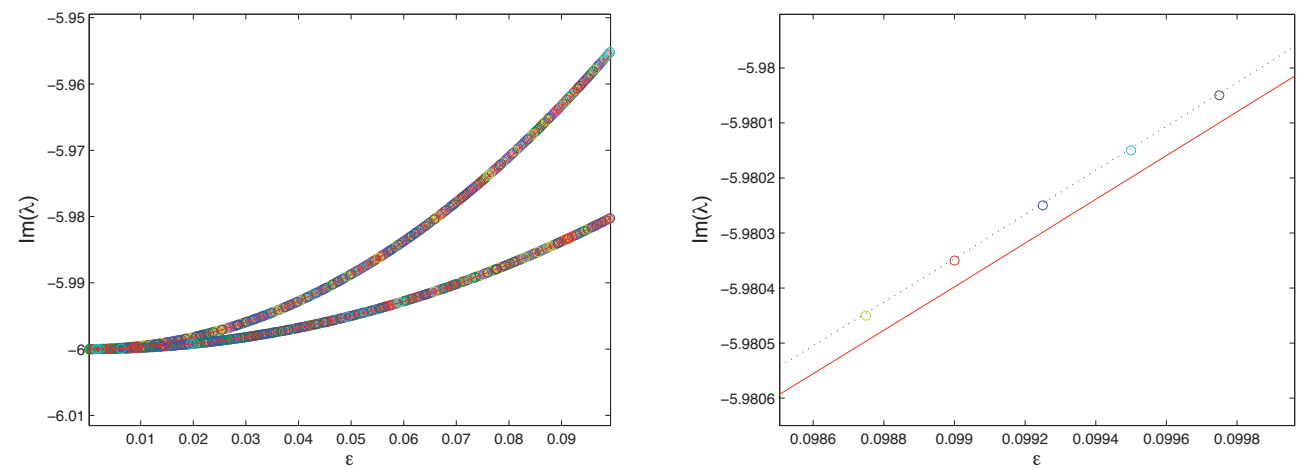

FIG. 4.1. The spectrum at $p=0$ of near the $\lambda_{0}=-6 i$. A DNS (see section 2.2) of the spectrum is marked with circles. The perturbative spectrum to $\mathcal{O}\left(\epsilon^{2}\right)$ is marked with solid red curves. The spectrum as computed by our new method (see section 3 ) to order $n=16$ is marked with dotted black curves. On the left, all three are essentially indistinguishable; on the right, a closer look at the upper curve for $0.0985 \leq \epsilon \leq 0.0999$ reveals the improvement of the higher-order approximation: The dotted curve closely approximates the circles.
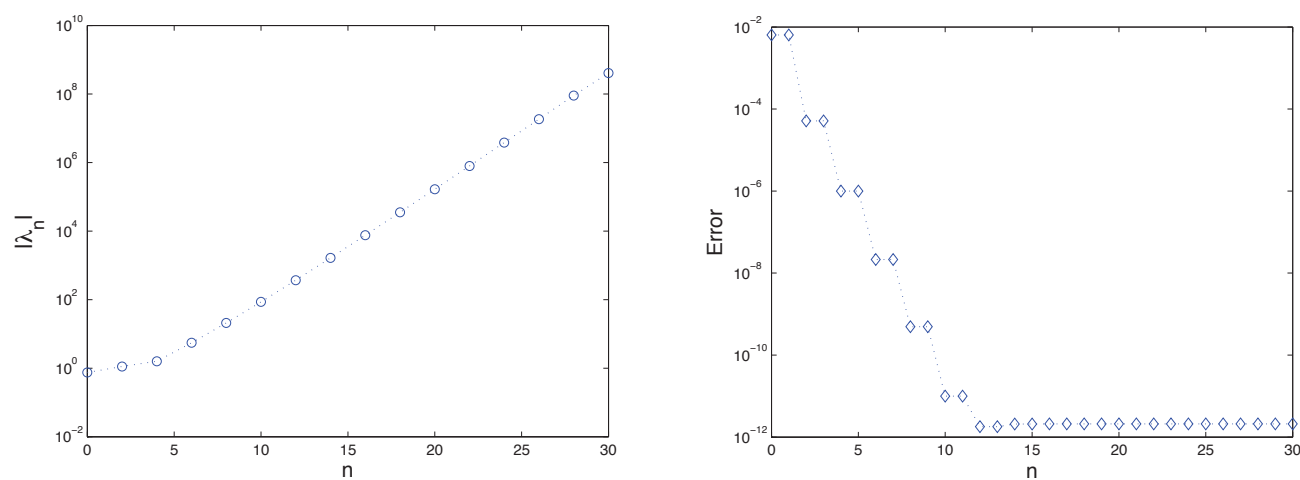

FIG. 4.2. The convergence of the TFE algorithm at an eigenvalue of multiplicity two is studied. Both panels take $p=0.25, \epsilon=0.075$, and consider an eigenvalue branch which bifurcates from $\lambda_{0}=-0.75 i$. Of the two branches which bifurcate from this $\lambda_{0}$, both panels are computed on the branch of (slightly) smaller magnitude. On the left, the magnitude of the corrections $\lambda_{n}$ is plotted as a function of $n$. The corrections are zero for odd $n$, as this is not a triad resonance, and are not plotted. On the right, a measure of the error, the difference between a DNS calculation of this eigenvalue and the TFE calculation, Error $=\left|\sum_{m=0}^{n} \lambda_{m} \epsilon^{m}-\lambda_{D N S}\right|$, is plotted as a function of the number of perturbation orders in the sum, $n$.

values are computed at $\epsilon=0.075$ on a branch which bifurcates from $\lambda_{0}=-0.75 i$ when $\epsilon=0$, with Bloch parameter $p=0.25$. The flat-state eigenvalue $\lambda_{0}=-0.75 i$ is an eigenvalue of multiplicity two; thus the convergence plots are a genuine test of the convergence of the extension of the previous algorithm for simple eigenvalues [9]. In Figure 4.2(left), the growth of the corrections $\lambda_{n}$ is observed as a function of $n$. In the right panel, the difference between a DNS calculation (see section 2.2) of this eigenvalue and the TFE calculation is plotted as a function of the number of perturbation orders included in the Taylor sum. Notice that this difference is approximately constant after $n=12$, suggesting that the $n=16$ in Figure 4.1 is more than sufficient 

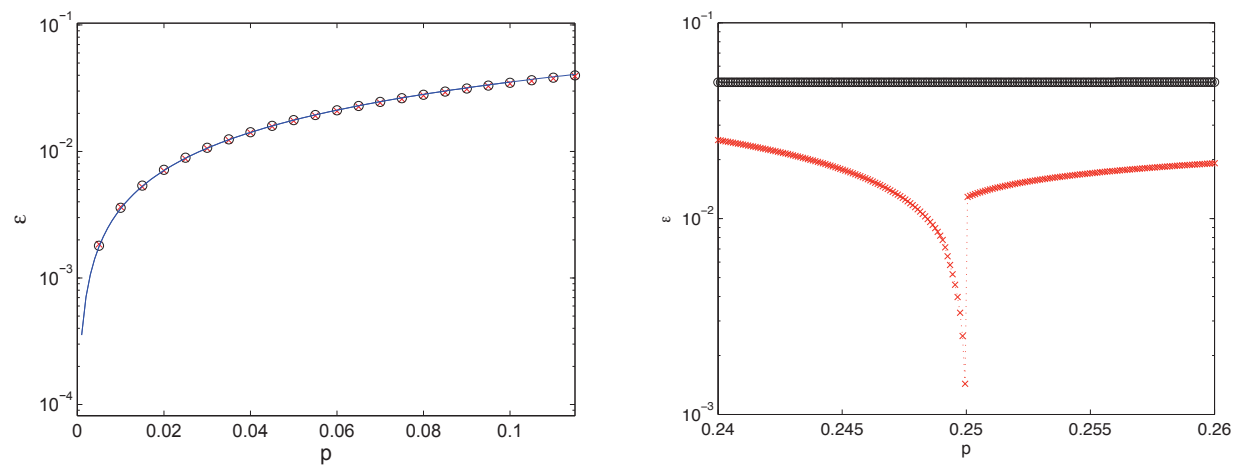

FIG. 4.3. The radius of convergence of the expansion is compared to the first value of $\epsilon$ where there is a crossing of eigenvalues. To compute the radius of convergence, the first nondegenerate pole of the Padé approximation of the series is computed, here marked with circles. The smallest value of $\epsilon$ where two eigenvalues collide is marked with $x$ 's. Left: For $p$ near 0 the series is immediately singular due to the Benjamin-Feir instability, marked with a solid curve. This curve agrees with the numerically computed first eigenvalue crossings and radii of convergence. Right: At $p=1 / 4$ there is a crossing at $\epsilon=0$, but the expansion converges until $\epsilon \approx 0.075$.

to resolve the spectrum.

CONJECTURe 1. All eigenvalues of multiplicity one or two for the spectral stability problem of periodic two-dimensional deep water waves are purely imaginary within the disk of analyticity of the spectral data, (3.2). More specifically, for $\lambda_{0}$ of multiplicity one or two we have

$$
\operatorname{Re}\left\{\lambda_{n}\right\}=0 \quad \forall n \geq 0 .
$$

We point out that this implies weak stability of periodic two-dimensional deep water traveling wavetrains to perturbations associated with eigenvalues of multiplicity one or two. This method does not take into account eigenvalues of multiplicity higher than two; however, for deep water waves on a two-dimensional fluid there is only one eigenvalue of multiplicity higher than two, $\lambda_{0}=0$ when $p=0$, from which the Benjamin-Feir instability arises. We now present a simple argument which precludes the analytic connection of the eigenvalues of the Benjamin-Feir instability to those of the flat state.

The Benjamin-Feir instability is a modulational instability of a plane wave to long wave disturbances corresponding to $p$ approaching zero. In the notation of this paper, the Benjamin-Feir instability for a Stokes wave (with wavenumber $k=1$ ) occurs for $p$ satisfying $0<p^{2}<8 \epsilon^{2}[28,30]$, which our numerics verify. There are two crucial observations with regard to the analyticity of the spectrum as a function of $\epsilon$ with $p$ varying. First, the instability does not include $p=0$ : Small amplitude deep water waves are stable to superharmonic perturbations [12, 18]. Second, for fixed small, but nonzero, $p$, when $\epsilon$ is increased across the Benjamin-Feir threshold the spectrum loses its analyticity. This loss of analyticity along the Benjamin-Feir threshold is plotted in Figure 4.3.

A limitation of our new approach, as with any perturbative method, is that it is valid only within the disk of convergence of the Taylor series. Furthermore, we have found (see [9]) that numerical analytic continuation schemes (e.g., Padé summation) are ineffective for this problem, indicating that the smallest singularity of the expansion lies on the real axis. We have found that this loss of analyticity 

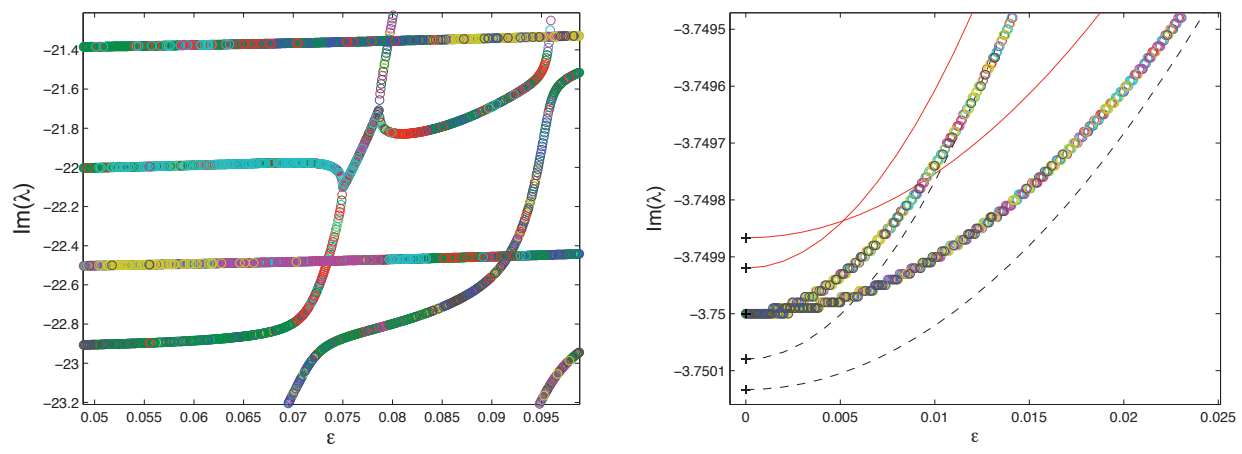

FIG. 4.4. Left: A DNS (see section 2.2) of the imaginary part of the spectrum as a function of wave slope, $\epsilon$. At intermediate wave slope, the spectrum loses analyticity in $\epsilon$, and branches of eigenvalues merge and bifurcate. This finite $\epsilon \approx 0.075$ crossing of eigenvalues results in a bubble of instability and a loss of analyticity of the spectrum. Right: Computed spectra near $\lambda_{0}=-3.75 i$ for $p=0.25$ (circles), $p=0.2499$ (solid lines), and 0.2501 (dotted lines). At $p=0.25$ there is a collision of eigenvalues at $\epsilon=0$, while for $p>0.25$ there is no collision for small $\epsilon$; however, for $p<0.25$ there is a small $\epsilon$ collision.

is a generic phenomenon for intermediate wave slopes and can occur at eigenvalue collisions, certainly when this collision leads to an eigenvalue leaving the imaginary axis. With this in mind, in Figure 4.3 we study the convergence properties of the perturbation series and note that eigenvalues may cross without loss of analyticity of the spectrum. In this figure we compare the smallest $\epsilon$ at which two eigenvalues cross to the disk of analyticity of the spectrum. We approximate the disk of analyticity by computing the first nondegenerate (i.e., noncancelled) pole of the Padé series, as in [9]. In the left panel we observe that, near $p=0$, there is both a collision of eigenvalues and loss of analyticity at $\epsilon=p / \sqrt{8}$, the Benjamin-Feir instability. In the right panel, there is loss of analyticity at approximately $\epsilon=0.075$, while there is a collision of eigenvalues of opposite Krein signature much sooner (at $\epsilon=0$ when $p=1 / 4$ ). In Figure 4.4 we present a simulation which exhibits many collisions of multiple eigenvalues. On the left we display a DNS of the spectrum at $p=1 / 4$, and we notice that there are multiple collisions where the spectrum remains analytic and there is no instability. The collision which does lead to instability, at approximately $(\epsilon \approx 0.075, \operatorname{Im}\{\lambda\} \approx-22.2)$, is of two eigenvalues which are not close at $\epsilon=0$. This is a finite amplitude instability which, interestingly, is unrelated to the two-dimensional null spaces at $p=1 / 4$ and $\epsilon=0$. That this instability persists at approximately the same value of $\epsilon$ as $p$ is varied accounts for the difference between the radius of convergence and the first crossing of eigenvalues in Figure 4.3. In the right panel of Figure 4.4 we observe that eigenvalues of opposite Krein signature collide for small $\epsilon$ when $p$ approaches $1 / 4$ from the left and do not collide at small $\epsilon$ for $p$ slightly larger than $1 / 4$, displaying a curious asymmetry with respect to the Bloch parameter which deserves further scrutiny.

5. Conclusion. In this paper we have presented a generalization of the transformed field expansions approach to perturbatively computing the spectrum of the linearized water wave operator $[8,9]$ to the crucially important case of eigenvalues of multiplicity two. We observe no instabilities which are analytically connected to the flat surface for deep water waves in the absence of surface tension. We present 
both a numerical method for computing the spectrum and an explicit computation of the first corrections to the flat-state eigenvalues of multiplicity two-a complete treatment of the analytic spectrum of deep water gravity waves on a two-dimensional fluid. Although the spectra computed here are all pure imaginary (thus the waves are weakly stable), such spectra still provide useful information about the traveling wave, for example as a basis from which to compute time periodic waves [21].

Ongoing research avenues include the extension of this algorithm to include surface tension and shallow water waves, where we expect the spectrum to include instabilities. The algorithm also simply extends to a three-dimensional fluid in the presence of three-dimensional perturbations, where other instabilities are known to occur. Although in principle the algorithm could be extended to higher-dimensional null spaces, these do not play a role in the two-dimensional deep water problem. Finally, a concurrent project is the analogous method for computing "Wilton ripples" and other traveling waves where the linear operator has null space of dimension larger than one.

Appendix A. Eigenvalues of multiplicity two. In this section we present the details of our generalization of the TFE approach to computing the spectrum of the linearized water wave operator to the case of an eigenvalue of algebraic and geometric multiplicity two. For notational convenience we present the method in the context of the generalized eigenvalue problem,

$$
(A+\lambda B) q=0
$$

(cf. (3.1)), where $A$ and $B$ depend analytically on $\epsilon$ and we suppose a priori that $v$ and $\lambda$ do as well. Expanding in Taylor series in $\epsilon$,

$$
A=\sum_{n=0}^{\infty} A_{n} \epsilon^{n}, \quad B=\sum_{n=0}^{\infty} B_{n} \epsilon^{n}, \quad \lambda=\sum_{n=0}^{\infty} \lambda_{n} \epsilon^{n}, \quad q=\sum_{n=0}^{\infty} q_{n} \epsilon^{n}
$$

(cf. (3.2)), and inserting these into the generalized eigenproblem (A.1) yields at leading order

$$
\left(A_{0}+\lambda_{0} B_{0}\right) q_{0}=0 .
$$

We presume throughout that $\operatorname{dim}\left(\operatorname{Ker}\left(A_{0}+\lambda_{0} B_{0}\right)\right)=2$, and that (A.3) has two independent solutions, $w_{1}$ and $w_{2}$, so that $q_{0}$ can be generically expressed as

$$
q_{0}=\alpha_{0} w_{1}+\beta_{0} w_{2}
$$

Furthermore, we assume that the null space of the adjoint of $\left(A_{0}+\lambda_{0} B_{0}\right)$ has two linearly independent solutions $\psi_{1}$ and $\psi_{2}$, so that, by the Fredholm alternative, the system

$$
\left(A_{0}+\lambda_{0} B_{0}\right) z=\theta
$$

has solutions if and only if

$$
\left\langle\psi_{1}, \theta\right\rangle=\left\langle\psi_{2}, \theta\right\rangle=0 .
$$

Expansion (A.2) transforms the nonlinear eigenvalue problem (A.1) into a series of linear equations at each order of $\epsilon$. Due to the two-dimensional null space associated 
with $\lambda_{0}$ there are two conditions (A.4) to be satisfied; however, as we shall see, there are two constants to be chosen at every order so that our new method proceeds smoothly from one order to the next. We observe that some of the constraints in (A.4) may be trivially satisfied, and to distinguish them one must carefully consider the inner products $\left\langle\psi_{j}, B_{0} w_{i}\right\rangle$ and $\left\langle\psi_{j}, A_{n} w_{i}\right\rangle$. The first simply tests the $B_{0}$-orthogonality of $\psi_{j}$ and $w_{i}$. The second vanishes unless the frequencies of $\psi_{j}, A_{n}$, and $w_{i}$ sum to zero, a resonance condition on the underlying wave and its perturbations. We will explore the cases $\left\langle\psi_{j}, A_{n} w_{i}\right\rangle \neq 0$ separately, classified by the first $n$ where such an inner product is nonzero.

A.1. Perturbation about triads. We begin with the simplest case:

$$
\left\langle\psi_{1}, B_{0} w_{2}\right\rangle=\left\langle\psi_{2}, B_{0} w_{1}\right\rangle=0, \quad\left\langle\psi_{2}, A_{1} w_{1}\right\rangle \neq 0 .
$$

The latter condition enforces a "triad interaction" as $\psi_{j} \sim e^{i \xi_{j} x}, w_{i} \sim e^{i \xi_{i} x}, A_{1} \sim$ $e^{i x}+$ c.c., where c.c. stands for complex conjugage, and $\left\langle\psi_{2}, A_{1} w_{1}\right\rangle \neq 0$ imply that the wavenumbers form a triad,

$$
\xi_{i}-\xi_{j} \pm 1=0
$$

(The temporal frequencies are also resonant, $\omega_{1}-\omega_{2} \pm 1=0$, for triad wavenumbers in a two-dimensional null space.) In this case, the $\mathcal{O}(\epsilon)$ equations are

$$
\left(A_{0}+\lambda_{0} B_{0}\right) q_{1}=-\left(A_{1}+\lambda_{1} B_{0}+\lambda_{0} B_{1}\right) q_{0},
$$

with solvability conditions

$$
\left(\begin{array}{cc}
\left\langle\psi_{1},\left(A_{1}+\lambda_{1} B_{0}+\lambda_{0} B_{1}\right) w_{1}\right\rangle & \left\langle\psi_{1},\left(A_{1}+\lambda_{1} B_{0}+\lambda_{0} B_{1}\right) w_{2}\right\rangle \\
\left\langle\psi_{2},\left(A_{1}+\lambda_{1} B_{0}+\lambda_{0} B_{1}\right) w_{1}\right\rangle & \left\langle\psi_{2},\left(A_{1}+\lambda_{1} B_{0}+\lambda_{0} B_{1}\right) w_{2}\right\rangle
\end{array}\right)\left(\begin{array}{c}
\alpha_{0} \\
\beta_{0}
\end{array}\right)=0
$$

or

$$
\begin{gathered}
\left(\begin{array}{rr}
\left\langle\psi_{1},\left(A_{1}+\lambda_{0} B_{1}\right) w_{1}\right\rangle & \left\langle\psi_{1},\left(A_{1}+\lambda_{0} B_{1}\right) w_{2}\right\rangle \\
\left\langle\psi_{2},\left(A_{1}+\lambda_{0} B_{1}\right) w_{1}\right\rangle & \left\langle\psi_{2},\left(A_{1}+\lambda_{0} B_{1}\right) w_{2}\right\rangle
\end{array}\right)\left(\begin{array}{c}
\alpha_{0} \\
\beta_{0}
\end{array}\right) \\
=-\lambda_{1}\left(\begin{array}{ll}
\left\langle\psi_{1}, B_{0} w_{1}\right\rangle & \left\langle\psi_{1}, B_{0} w_{2}\right\rangle \\
\left\langle\psi_{2}, B_{0} w_{1}\right\rangle & \left\langle\psi_{2}, B_{0} w_{2}\right\rangle
\end{array}\right)\left(\begin{array}{c}
\alpha_{0} \\
\beta_{0}
\end{array}\right) .
\end{gathered}
$$

From these conditions we determine the $\lambda_{1}$ from a quadratic equation (the determinant of the $2 \times 2$ matrix in (A.6) set to zero) and the relationship between $\alpha$ and $\beta_{0}$ as they are a generalized eigenvector of each eigenvalue $\lambda_{1},($ A.7). Without loss of generality we will choose $\alpha=1$ at order zero, and $\alpha=0$ at subsequent orders so that $w_{1}$ will not appear in the homogeneous solutions at later orders of the perturbation solution. The value $\beta_{0}$ is the leading-order term in the ratio of $w_{1}$ to $w_{2}$; the full ratio $\beta$ is corrected at higher orders. Whether $\beta_{0}$ is determined here or later depends on the null space of the matrix in (A.6).

The first correction of the eigenfunction can be expressed as

$$
q_{1}=q_{1, p}+q_{1, h}
$$

where $q_{1, p}$ is a particular solution to (A.5),

$$
q_{1, p}=-\left(A_{0}+\lambda_{0} B_{0}\right)^{-1}\left(A_{1}+\lambda_{1} B_{0}+\lambda_{0} B_{1}\right) q_{0},
$$

Copyright $@$ by SIAM. Unauthorized reproduction of this article is prohibited. 
and the inverse is taken over the complement of the null space of $\left(A_{0}+\lambda_{0} B_{0}\right)$. An arbitrary amplitude homogeneous solution is also included, $q_{1, h}=\beta_{1} w_{2}$ (no contribution of $w_{1}$ is necessary because of the definition of $\alpha$ ). Continuing to $\mathcal{O}\left(\epsilon^{2}\right)$,

$$
\left(A_{0}+\lambda_{0} B_{0}\right) q_{2}=-\left(A_{1}+\lambda_{1} B_{0}+\lambda_{0} B_{1}\right) q_{1}-\left(A_{2}+\lambda_{2} B_{0}+\lambda_{1} B_{1}\right) q_{0},
$$

which implies the solvability condition

$$
\begin{gathered}
\left(\begin{array}{cc}
\left\langle\psi_{1},\left(A_{1}+\lambda_{1} B_{0}+\lambda_{0} B_{1}\right) w_{2}\right\rangle & \left\langle\psi_{1}, B_{0} v_{0}\right\rangle \\
\left\langle\psi_{2},\left(A_{1}+\lambda_{1} B_{0}+\lambda_{0} B_{1}\right) w_{2}\right\rangle & \left\langle\psi_{2}, B_{0} v_{0}\right\rangle
\end{array}\right)\left(\begin{array}{c}
\beta_{1} \\
\lambda_{2}
\end{array}\right) \\
=-\left(\begin{array}{l}
\left\langle\psi_{1},\left(A_{1}+\lambda_{1} B_{0}+\lambda_{0} B_{1}\right) q_{1, p}+\left(A_{2}+\lambda_{1} B_{1}\right) q_{0}\right\rangle \\
\left\langle\psi_{2},\left(A_{1}+\lambda_{1} B_{0}+\lambda_{0} B_{1}\right) q_{1, p}+\left(A_{2}+\lambda_{1} B_{1}\right) q_{0}\right\rangle
\end{array}\right)
\end{gathered}
$$

which determines $\beta_{1}$ and $\lambda_{2}$. The general $n$ th-order term is similar to the $\mathcal{O}\left(\epsilon^{2}\right)$ equation

$$
\left(A_{0}+\lambda_{0} B_{0}\right) q_{n}=-\sum_{j=1}^{n}\left(A_{j}+\sum_{k=0}^{j} \lambda_{k} B_{j-k}\right) q_{n-j},
$$

which implies the solvability conditions

$$
\begin{aligned}
& \left(\begin{array}{cc}
\left\langle\psi_{1},\left(A_{1}+\lambda_{1} B_{0}+\lambda_{0} B_{1}\right) w_{2}\right\rangle & \left\langle\psi_{1}, B_{0} q_{0}\right\rangle \\
\left\langle\psi_{2},\left(A_{1}+\lambda_{1} B_{0}+\lambda_{0} B_{1}\right) w_{2}\right\rangle & \left\langle\psi_{2}, B_{0} q_{0}\right\rangle
\end{array}\right)\left(\begin{array}{c}
\beta_{n-1} \\
\lambda_{n}
\end{array}\right)
\end{aligned}
$$

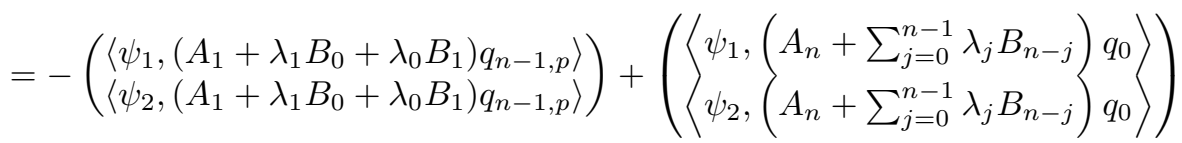

$$
\begin{aligned}
& +\left(\left\langle\begin{array}{l}
\left\langle\psi_{1}, \sum_{j=2}^{n-1}\right. \\
\left\langle\psi_{2}, \sum_{j=2}^{n-1}\left(A_{j}+\sum_{k=0}^{j} \lambda_{k} B_{j-k}\right.\right.
\end{array}\left(\begin{array}{l}
A_{j}+\sum_{k=0}^{j} \lambda_{k} B_{j-k}
\end{array}\right) \begin{array}{l}
q_{n-j} \\
q_{n-j}
\end{array}\right) .\right.
\end{aligned}
$$

How one explicitly computes the $q_{n-1, p}$ depends on the basis chosen for the original linear problem. A key point here is that the matrix that we must invert does not change at each order. Although it is not the leading-order matrix, it is only the next order, and the formal existence of solutions is conditional only on the invertibility of this matrix.

The above procedure relies on $\beta_{0}$ being determined at second order. This requires, at least, that elements in the null space of $\left(A_{0}+\lambda_{0} B_{0}\right)$ have wavenumbers which satisfy $\xi_{1}-\xi_{2} \pm 1=0$ and $\omega_{1}-\omega_{2} \pm 1=0$, a triad interaction. Even if the frequencies sum to zero, there may be additional cancellation in the inner products, which must be handled on a case-by-case basis. If the $\xi_{j}$ do not satisfy such a triad interaction, or if the matrix in (A.8) is singular due to other cancellations, the procedure of this section requires modification. It is well known that deep water gravity waves do not exhibit triad interactions [28]; thus another expansion will be necessary for deep water gravity waves.

A.2. Perturbation about quartets. For the generic choice of $\xi_{1}$ and $\xi_{2}$, many of the previously presented inner products are zero. For instance, if $\left|\xi_{2}-\xi_{1}\right|>1$, then the leading-order equation (cf. (A.6)) is

$$
\left(\begin{array}{cc}
\lambda_{1}\left\langle\psi_{1}, B_{0} w_{1}\right\rangle & 0 \\
0 & \lambda_{1}\left\langle\psi_{2}, B_{0} w_{2}\right\rangle
\end{array}\right)\left(\begin{array}{c}
1 \\
\beta_{0}
\end{array}\right)=0
$$

Copyright (c) by SIAM. Unauthorized reproduction of this article is prohibited. 
because $\left\langle\psi_{i}, A_{1} w_{j}\right\rangle=\left\langle\psi_{i}, B_{1} w_{j}\right\rangle=0$ (both $A_{1}$ and $B_{1}$ are supported only at frequency $\xi= \pm 1)$. This implies $\lambda_{1}=0$ and leaves $\beta_{0}$ arbitrary. In this case the $\mathcal{O}\left(\epsilon^{2}\right)$ solvability conditions are

$$
\begin{aligned}
& \left\langle\psi_{1},\left(A_{1}+\lambda_{0} B_{1}\right) q_{1, p}+\left(A_{2}+\lambda_{2} B_{0}+\lambda_{0} B_{2}\right) q_{0}\right\rangle=0, \\
& \left\langle\psi_{2},\left(A_{1}+\lambda_{0} B_{1}\right) q_{1, p}+\left(A_{2}+\lambda_{2} B_{0}+\lambda_{0} B_{2}\right) q_{0}\right\rangle=0,
\end{aligned}
$$

which leaves $\beta_{1}$ arbitrary (the inner products $\left\langle\psi_{j},\left(A_{1}+\lambda_{0} B_{1}\right) w_{2}\right\rangle=0$ without triads) and results in a (possibly nonlinear) system for $\beta_{0}$ and $\lambda_{2}$.

Moving beyond the triad resonances, we begin with the quartet resonance (i.e., $\left.\xi_{2}-\xi_{1} \pm 2=0\right)$ in this section and consider the higher-order case $\left(\left|\xi_{1}-\xi_{2}\right|>2\right)$ in subsection A.3. If we recall that

$$
\begin{aligned}
q_{1, p}= & -\left(A_{0}+\lambda_{0} B_{0}\right)^{-1}\left(A_{1}+\lambda_{1} B_{0}+\lambda_{0} B_{1}\right) q_{0} \\
= & -\left(A_{0}+\lambda_{0} B_{0}\right)^{-1}\left(A_{1}+\lambda_{1} B_{0}+\lambda_{0} B_{1}\right) w_{1} \\
& -\beta_{0}\left(A_{0}+\lambda_{0} B_{0}\right)^{-1}\left(A_{1}+\lambda_{1} B_{0}+\lambda_{0} B_{1}\right) w_{2},
\end{aligned}
$$

then (A.10) is of the form

$$
\lambda_{2}+P_{11}+\beta_{0} P_{12}=0, \quad \beta_{0} \lambda_{2}+\beta_{0} P_{22}+P_{21}=0,
$$

with

$$
\begin{aligned}
& P_{11}=\frac{\left\langle\psi_{1},\left(A_{2}+\lambda_{0} B_{2}\right) w_{1}-\left(A_{1}+\lambda_{0} B_{1}\right)\left(A_{0}+\lambda_{0} B_{0}\right)^{-1}\left(A_{1}+\lambda_{1} B_{0}\right) w_{1}\right\rangle}{\left\langle\psi_{1}, B_{0} w_{1}\right\rangle}, \\
& P_{12}=\frac{\left\langle\psi_{1},\left(A_{2}+\lambda_{0} B_{2}\right) w_{2}-\left(A_{1}+\lambda_{0} B_{1}\right)\left(A_{0}+\lambda_{0} B_{0}\right)^{-1}\left(A_{1}+\lambda_{1} B_{0}\right) w_{2}\right\rangle}{\left\langle\psi_{1}, B_{0} w_{1}\right\rangle}, \\
& P_{21}=\frac{\left\langle\psi_{2},\left(A_{2}+\lambda_{0} B_{2}\right) w_{1}-\left(A_{1}+\lambda_{0} B_{1}\right)\left(A_{0}+\lambda_{0} B_{0}\right)^{-1}\left(A_{1}+\lambda_{1} B_{0}\right) w_{1}\right\rangle}{\left\langle\psi_{2}, B_{0} w_{2}\right\rangle}, \\
& P_{22}=\frac{\left\langle\psi_{2},\left(A_{2}+\lambda_{0} B_{2}\right) w_{2}-\left(A_{1}+\lambda_{0} B_{1}\right)\left(A_{0}+\lambda_{0} B_{0}\right)^{-1}\left(A_{1}+\lambda_{1} B_{0}\right) w_{2}\right\rangle}{\left\langle\psi_{2}, B_{0} w_{2}\right\rangle} .
\end{aligned}
$$

Writing (A.12) in terms of $\lambda_{2}$ alone delivers

$$
\lambda_{2}^{2}+\left(P_{11}+P_{22}\right) \lambda_{2}+\left(P_{11} P_{22}-P_{12} P_{21}\right)=0,
$$

so that $\lambda_{2}$ and, from (A.12), $\beta_{0}$ can be determined. Recall that $\lambda_{0}$ has already been chosen, and $\lambda_{1}=0 ; \beta_{1}$ is still arbitrary. At later orders the equations become

$$
M\left(\begin{array}{c}
\beta_{n-2} \\
\lambda_{n}
\end{array}\right)=R
$$

Copyright $@$ by SIAM. Unauthorized reproduction of this article is prohibited. 
where

$$
\begin{aligned}
& M=\left(\begin{array}{cc}
\left\langle\psi_{1},\left(A_{2}+\lambda_{2} B_{0}+\lambda_{0} B_{2}\right) w_{2}\right\rangle & \left\langle\psi_{1}, B_{0} w_{1}\right\rangle \\
\left\langle\psi_{2},\left(A_{2}+\lambda_{2} B_{0}+\lambda_{0} B_{2}\right) w_{2}\right\rangle & \beta_{0}\left\langle\psi_{2}, B_{0} w_{2}\right\rangle
\end{array}\right) \\
& +\left(\begin{array}{lll}
\left\langle\psi_{1},-\left(A_{1}+\lambda_{0} B_{1}\right)\left(A_{0}+\lambda_{0} B_{0}\right)^{-1}\left(A_{1}+\lambda_{1} B_{0}\right) w_{2}\right\rangle & 0 \\
\left\langle\psi_{2},-\left(A_{1}+\lambda_{0} B_{1}\right)\left(A_{0}+\lambda_{0} B_{0}\right)^{-1}\left(A_{1}+\lambda_{1} B_{0}\right) w_{2}\right\rangle & 0
\end{array}\right), \\
& \left.R=-\left(\begin{array}{l}
\left\langle\psi_{1}, \sum_{j=1}^{2}\right. \\
\psi_{2}, \sum_{j=1}^{2}
\end{array}\left(\begin{array}{l}
A_{j}+\sum_{k=0}^{j} \lambda_{j-k} B_{k} \\
A_{j}+\sum_{k=0}^{j} \lambda_{j-k} B_{k}
\end{array}\right) q_{n-j, p}\right\rangle\right) \\
& -\left(\begin{array}{l}
\left\langle\psi_{1},\left(A_{n}+\sum_{k=1}^{n} \lambda_{n-k} B_{k}\right)\right. \\
\left\langle\psi_{2},\left(A_{n}+\sum_{k=1}^{n} \lambda_{n-k} B_{k}\right.\right.
\end{array}\right) \\
& \left.-\left(\begin{array}{l}
\left\langle\psi_{1}, \sum_{j=3}^{n-1}\right. \\
\psi_{2}, \sum_{j=3}^{n-1}
\end{array}\left(\begin{array}{l}
A_{j}+\sum_{k=0}^{j} \lambda_{j-k} B_{k} \\
A_{j}+\sum_{k=0}^{j} \lambda_{j-k} B_{k}
\end{array}\right) q_{n-j}\right\rangle\right) .
\end{aligned}
$$

As before, the equation for the leading correction $\beta_{0}$ is at most quadratic; all higherorder corrections $\beta_{j}$ are determined by linear equations. For deep water gravity waves the quartet resonance has $P_{12}=P_{21}=0$, so that (A.12) becomes

$$
\lambda_{2}+P_{11}=0, \quad \beta_{0} \lambda_{2}+\beta_{0} P_{22}=0,
$$

which has solutions $\lambda_{2}=-P_{11}$ and $\beta_{0}=0$. Furthermore, in this case we can simplify (A.13) to

$$
\begin{aligned}
& \text { (A.14) } \quad\left(\begin{array}{cc}
0 & \left\langle\psi_{1}, B_{0} w_{1}\right\rangle \\
\left(\lambda_{2}\left(\xi_{2}\right)-\lambda_{2}\left(\xi_{1}\right)\right)\left\langle\psi_{2}, B_{0} w_{2}\right\rangle & 0
\end{array}\right)\left(\begin{array}{c}
\beta_{n-2} \\
\lambda_{n}
\end{array}\right)
\end{aligned}
$$

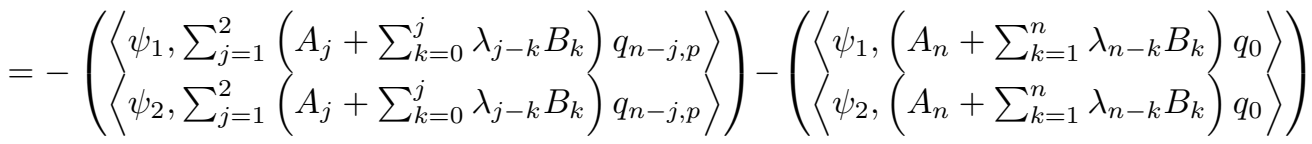

$$
\begin{aligned}
& \left.\left.-\left(\begin{array}{l}
\left\langle\psi_{1}, \sum_{j=3}^{n-1}\left(A_{j}+\sum_{k=0}^{j} \lambda_{j-k} B_{k}\right) q_{n-j}\right\rangle \\
\left\langle\psi_{2}, \sum_{j=3}^{n-1}\left(A_{j}+\sum_{k=0}^{j} \lambda_{j-k} B_{k}\right.\right.
\end{array}\right) . q_{n-j}\right\rangle\right) .
\end{aligned}
$$

Here $\lambda_{2}\left(\xi_{j}\right)=-P_{j j}$, the correction $\lambda_{2}$ given by (A.12) if $w_{1}$ is supported at wavenumber $\xi_{j}$. Notice that the solvability of (A.14) depends only on the difference $\lambda_{2}\left(\xi_{2}\right)-\lambda_{2}\left(\xi_{1}\right)$. In the previous section we explicitly computed $\lambda_{2}\left(\xi_{j}\right)$ in deep water. They are distinct; thus we may formally compute $\lambda_{n}$ and $\beta_{n}$ to all orders.

A.3. Perturbation about higher degree resonances. If the resonance is higher order than a triad or quartet, the $\mathcal{O}\left(\epsilon^{2}\right)$ solvability conditions yield a linear equation for $\lambda_{2}$,

$$
\lambda_{2}=-\frac{\left\langle\psi_{1},\left(A_{1}+\lambda_{0} B_{1}\right) q_{1, p}+\left(A_{2}+\lambda_{0} B_{2}\right) w_{1}\right\rangle}{\left\langle\psi_{1}, B_{0} w_{1}\right\rangle},
$$

which in fact gives two eigenvalues. If we label so that $w_{1}$ is supported at frequency $\xi_{1}$, this gives $\lambda_{2}\left(\xi_{1}\right)$, and, relabelling, gives $\lambda_{2}\left(\xi_{2}\right)$. The corresponding equation for $\beta_{0}$ is

$$
\beta_{0}\left(\lambda_{2}\left(\xi_{1}\right)-\lambda_{2}\left(\xi_{2}\right)\right)=0
$$

Copyright (c) by SIAM. Unauthorized reproduction of this article is prohibited. 
which implies $\beta_{0}=0$. (The $\lambda_{2}$ are computed explicitly in the previous subsection and are distinct.)

The solvability conditions at $\mathcal{O}\left(\epsilon^{3}\right)$ are

$$
\begin{aligned}
& \left\langle\psi_{1},\left(A_{3}+\lambda_{3} B_{0}+\lambda_{2} B_{1}+\lambda_{0} B_{3}\right) q_{0}+\left(A_{2}+\lambda_{0} B_{2}+\lambda_{2} B_{0}\right) q_{1}+\left(A_{1}+\lambda_{0} B_{1}\right) q_{2}\right\rangle=0, \\
& \left\langle\psi_{2},\left(A_{3}+\lambda_{3} B_{0}+\lambda_{2} B_{1}+\lambda_{0} B_{3}\right) q_{0}+\left(A_{2}+\lambda_{0} B_{2}+\lambda_{2} B_{0}\right) q_{1}+\left(A_{1}+\lambda_{0} B_{1}\right) q_{2}\right\rangle=0 .
\end{aligned}
$$

Here $\beta_{1}, \beta_{2}$, and $\lambda_{3}$ are unknowns. The inner products which involve $\beta_{2}$ are zero; thus this system is linear for $\beta_{1}$ and $\lambda_{3}$ :

$$
\begin{gathered}
\left(\begin{array}{cc}
0 & \left\langle\psi_{1}, B_{0} w_{1}\right\rangle \\
\left(\lambda_{2}\left(\xi_{2}\right)-\lambda_{2}\left(\xi_{1}\right)\right)\left\langle\psi_{2}, B_{0} w_{2}\right\rangle & 0
\end{array}\right)\left(\begin{array}{c}
\beta_{1} \\
\lambda_{3}
\end{array}\right) \\
=-\left(\begin{array}{c}
\left\langle\psi_{1},\left(A_{3}+\lambda_{2} B_{1}+\lambda_{0} B_{3}\right) q_{0}\right\rangle \\
\left\langle\psi_{2},\left(A_{3}+\lambda_{2} B_{1}+\lambda_{0} B_{3}\right) q_{0}\right\rangle
\end{array}\right) \\
-\left(\begin{array}{c}
\left\langle\psi_{1},\left(A_{2}+\lambda_{0} B_{2}+\lambda_{2} B_{0}\right) q_{1, p}\right\rangle \\
\left\langle\psi_{2},\left(A_{2}+\lambda_{0} B_{2}+\lambda_{2} B_{0}\right) q_{1, p}\right\rangle
\end{array}\right)-\left(\begin{array}{c}
\left\langle\psi_{1},\left(A_{1}+\lambda_{0} B_{1}\right) q_{2, p}\right\rangle \\
\left\langle\psi_{2},\left(A_{1}+\lambda_{0} B_{1}\right) q_{2, p}\right\rangle
\end{array}\right) .
\end{gathered}
$$

Higher orders follow this pattern, with $\beta_{n-2}$ and $\lambda_{n}$ determined at order $n$, e.g.,

$$
\begin{aligned}
& -\left(\left\langle\psi_{1}, B_{0} w_{1}\right\rangle\right) \lambda_{n}=\left\langle\psi_{1}, \sum_{j=1}^{2}\left(A_{j}+\sum_{k=0}^{j} \lambda_{k} B_{j-k}\right) q_{n-j, p}\right\rangle \\
& +\left\langle\psi_{1}, \sum_{j=3}^{n-1}\left(A_{j}+\sum_{k=0}^{j} \lambda_{k} B_{j-k}\right) q_{n-j}\right\rangle+\left\langle\psi_{1},\left(A_{n}+\sum_{k=0}^{n-1} \lambda_{k} B_{n-k}\right) q_{0}\right\rangle
\end{aligned}
$$

and

$$
\begin{gathered}
-\left(\lambda_{2}\left(\xi_{1}\right)-\lambda_{2}\left(\xi_{2}\right)\right)\left\langle\psi_{2}, B_{0} w_{2}\right\rangle \beta_{n-2}=\left\langle\psi_{1}, \sum_{j=1}^{2} A_{j}+\sum_{k=0}^{j} \lambda_{k} B_{j-k} q_{n-j, p}\right\rangle \\
+\left\langle\psi_{1}, \sum_{j=3}^{n-1}\left(A_{j}+\sum_{k=0}^{j} \lambda_{k} B_{j-k}\right) q_{n-j}\right\rangle+\left\langle\psi_{1},\left(A_{n}+\sum_{k=0}^{n-1} \lambda_{k} B_{n-k}\right) q_{0}\right\rangle .
\end{gathered}
$$

In the numerical method of this paper, all inner products are computed spectrally using Fourier collocation for the horizontal direction and a Chebyshev-Tau method for the vertical; see [7] for details.

\section{REFERENCES}

[1] G. G. Stokes, On the theory of oscillatory waves, Trans. Camb. Phil. Soc., 8 (1847), pp. 441455.

[2] W. J. HARrison, The influence of viscosity and capillarity on waves of finite amplitude, Proc. Lond. Math. Soc., 7 (1909), pp. 107-121.

[3] R. J. C. Kamesvara, On ripples of finite amplitude, Proc. Indian Ass. Cultiv. Sci., 6 (1920), pp. $175-193$.

[4] H. Lamb, Hydrodynamics, Cambridge University Press, Cambridge, UK, 1879.

[5] L. F. MCGOLDRICK, An experiment on second-order capillary gravity resonant wave interactions, J. Fluid Mech., 40 (1970), pp. 251-271.

[6] J. Reeder And M. Shinbrot, Three dimensional, nonlinear wave interaction in water of constant depth, Nonlinear Anal., 5 (1981), pp. 303-323.

Copyright (c) by SIAM. Unauthorized reproduction of this article is prohibited. 
[7] D. P. Nicholls And F. Reitich, Stable, high-order computation of traveling water waves in three dimensions, Eur. J. Mech. B Fluids, 25 (2006), pp. 406-424.

[8] D. P. Nicholls, Spectral stability of traveling water waves: Analytic dependence of the spectrum, J. Nonlinear Sci., 17 (2007), pp. 369-397.

[9] D. P. Nicholls, Spectral data for traveling water waves: Singularities and stability, J. Fluid Mech., 624 (2009), pp. 339-360.

[10] D. P. Nicholls, Spectral stability of traveling water waves: Eigenvalue collision, singularities, and direct numerical simulation, Phys. D, 240 (2011), pp. 376-381.

[11] B. Deconinck And J. N. Kutz, Computing spectra of linear operators using the FloquetFourier-Hill method, J. Comput. Phys., 219 (2006), pp. 296-321.

[12] M. S. Longuet-Higgins, The instabilities of gravity waves of finite amplitude in deep water. I. Superharmonics, Proc. Roy. Soc. London Ser. A, 360 (1978), pp. 471-488.

[13] M. S. Longuet-Higgins, The instabilities of gravity waves of finite amplitude in deep water. II. Subharmonics, Proc. Roy. Soc. London Ser. A, 360 (1978), pp. 489-505.

[14] M. Francius And C. Kharif, Three-dimensional instabilities of periodic gravity waves in shallow water, J. Fluid Mech., 561 (2006), pp. 417-437.

[15] B. DeConinck And K. Oliveras, The instability of periodic surface gravity waves, J. Fluid Mech., 675 (2011), p. 141-167.

[16] F. Dias And C. Kharif, Nonlinear gravity and gravity-capillary waves, in Annu. Rev. Fluid Mech. 31, Annual Reviews, Palo Alto, CA, 1999, pp. 301-346.

[17] D. P. Nicholls And F. Reitich, On analyticity of traveling water waves, Proc. Roy. Soc. London Ser. A, 461 (2005), pp. 1283-1309.

[18] R. S. Mackay and P. G. Saffman, Stability of water waves, Proc. Roy. Soc. London Ser. A, 406 (1986), pp. 115-125.

[19] V. Zakharov, Stability of periodic waves of finite amplitude on the surface of a deep fluid, J. Appl. Mech. Tech. Phys., 9 (1968), pp. 190-194.

[20] J. MCLEAn, Instabilities of finite-amplitude gravity waves on water of finite depth, J. Fluid Mech., 114 (1982), pp. 331-341.

[21] D. M. Ambrose and J. Wilkening, Computation of time-periodic solutions of the BenjaminOno equation, J. Nonlinear Sci., 20 (2010), pp. 277-308.

[22] B. Akers AND D. P. Nicholls, Traveling waves in deep water with gravity and surface tension, SIAM J. Appl. Math., 70 (2010), pp. 2373-2389.

[23] N. A. Phillips, A coordinate system having some special advantages for numerical forecasting, J. Atmospheric Sci., 14 (1957), pp. 184-185.

[24] J. Chandezon, D. Maystre, and G. Raoult, A new theoretical method for diffraction gratings and its numerical application, J. Opt., 11 (1980), pp. 235-241.

[25] W. Craig and C. Sulem, Numerical simulation of gravity waves, J. Comput. Phys., 108 (1993), pp. $73-83$.

[26] E. ButKov, Mathematical Physics, Addison-Wesley, Reading, MA, 1968.

[27] J. P. Keener, Principles of Applied Mathematics, Perseus Books, New York, 2000.

[28] A. D. D. Craik, Wave Interactions and Fluid Flows, Cambridge University Press, Cambridge, UK, 1985.

[29] B. AKERs, The generation of capillary-gravity solitary waves by a surface pressure forcing, Math. Comp. Simul., 82 (2012), pp. 958-967.

[30] B. Akers And P. A. Milewski, Dynamics of three dimensional gravity-capillary solitary waves in deep water, SIAM J. Appl. Math., 70 (2010), pp. 2390-2408.

[31] M. J. Ablowitz, A. S. Fokas, and Z. H. Musslimani, On a new non-local formulation of water waves, J. Fluid Mech., 562 (2006), pp. 313-343.

[32] D. P. Nicholls, Efficient enforcement of the far-field boundary conditions in the transformed field expansion method, J. Comput. Phys., 230 (2011), pp. 8290-8303.

Copyright (c) by SIAM. Unauthorized reproduction of this article is prohibited. 